\title{
Gravidez decorrente de violência sexual: Caracterização sociodemográfica das mulheres e desfechos do aborto em um serviço público de referência, São Paulo, Brasil
}

\section{Pregnancy resulting from sexual violence: Socio-demographic characterization of women and outcomes of abortion in a public service of reference, São Paulo, Brazil}

\author{
DOI: $10.46814 /$ lajdv3n3-035
}

Recebimento dos originais: 01/052021

Aceitação para publicação: 31/06/2021

\author{
Daniela Pedroso \\ Mestrado em Saúde Materno Infantil pela Universidade Santo Amaro \\ Núcleo de Violência Sexual e Aborto Legal - Hospital Pérola Byington \\ Avenida Brigadeiro Luiz Antônio 683. Bela Vista, São Paulo (SP), Brasil - CEP 01317-000 \\ E-mail: dani.pedroso@uol.com.br
}

\section{Yára Juliano}

Doutorado em Distúrbios da Comunicação Humana pela Universidade Federal de São Paulo Departamento de Saúde Coletiva. Universidade Santo Amaro

Rua Prof. Enéas de Siqueira Neto 340. Jardim das Imbuias, São Paulo (SP), Brasil - CEP 04829-300

E-mail: yjuliano@prof.unisa.br

\section{Patrícia Colombo de Souza}

Doutorado em Nutrição em Saúde Pública pela Universidade Federal de São Paulo

Departamento de Saúde Coletiva. Universidade Santo Amaro

Rua Prof. Enéas de Siqueira Neto 340. Jardim das Imbuias, São Paulo (SP), Brasil - CEP 04829-300

E-mail: pcolombo@prof.unisa.br

\section{Jefferson Drezett}

Doutorado em Ciências da Saúde pela Faculdade de Medicina do ABC

Faculdade de Saúde Pública da Universidade de São Paulo

Avenida Dr. Arnaldo 715. Cerqueira César, São Paulo (SP), Brasil - CEP 01246-904

E-mail: drezett@usp.br

\section{RESUMO}

Introdução: A restritiva legislação brasileira permite o aborto em casos excepcionais, como na gravidez decorrente de crime sexual. Entretanto, poucos serviços de saúde oferecem atendimento e pouco se conhece sobre os desfechos do aborto para essas mulheres. Objetivo: Analisar características sociodemográficas de mulheres com gravidez decorrente de violência sexual e fatores associados ao aborto. Método: Estudo transversal com amostra de conveniência de 2.418 mulheres com alegação de gravidez decorrente de violência sexual atendidas no Hospital Pérola Byington de agosto/1994 a dezembro/2017. As variáveis de estudo foram analisadas segundo realizar ou não o aborto. Considerouse idade, escolaridade, raça/cor, situação união, religião, ocupação, intimidação, agressor, realização de boletim de ocorrência e exame médico-legal. Os dados foram analisados em Epi Info 7.2.3.1, por meio de Qui-quadrado de Pearson e teste de Mann Whitney para amostras independentes, com $p<0,05$ e Intervalo de Confiança de 95\%. Aprovado pelo Comitê de Ética em Pesquisa do Hospital Pérola Byington, protocolo $\mathrm{n}^{\circ}$ 046/11. Resultados: Mulheres que não realizaram o aborto apresentaram 
menor média de idade do que as que realizaram o procedimento (22,9 $\pm 4,5$ anos versus $25,4 \pm 7,2$ anos),

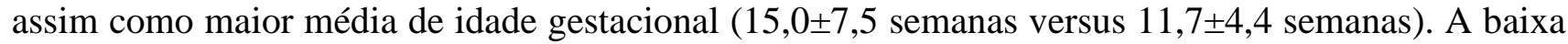
escolaridade, não ter ocupação, agressor conhecido, grave ameaça, não realizar boletim de ocorrência policial e não realizar exame médico-legal foi mais frequente nas mulheres que não realizaram o aborto. Entre os motivos para não realizar o aborto a desistência da mulher foi o mais frequente $(25,0 \%)$, seguido de idade gestacional $\geq 23$ semanas $(23,1 \%)$. Conclusão: A desistência da mulher e a idade gestacional avançada foram as principais razões para não realizar o aborto legal. Os achados sugerem que a maior vulnerabilidade de mulheres mais jovens, sem renda e com pouca escolaridade termine como obstáculo. Agressores conhecidos e que utilizam de ameaças parecem exercer alguma influência nesses casos, possivelmente postergando a busca pelo aborto legal.

Palavras-chave: Aborto Legal, Gravidez não desejada, Violência sexual, Violência contra a mulher, Vítimas de crime.

\begin{abstract}
Background: The restrictive Brazilian criminal law allows abortion in exceptional cases, such as pregnancy resulting from sexual crime. However, few health services offer care and little is known about these women and abortion outcomes. Objective: To analyze sociodemographic characteristics of women with pregnancy due to sexual violence and factors associated with abortion. Method: Crosssectional study with a convenience sample of 2,418 women with allegations of pregnancy due to sexual violence attended at Pérola Byington Hospital, August/1994 to December/2017. The study variables were analyzed according to performing abortion or not. Age, schooling, race/color, union situation, religion, occupation, intimidation, aggressor, police report and medical-legal examination were considered. The data were analyzed in Epi Info 7.2.3.1, using Pearson's Chi-square and Mann Whitney test for independent samples, with $\mathrm{p}<0.05$ and 95\% Confidence Interval. Approved by the Research Ethics Committee, Pérola Byington Hospital, Protocol 046/11. Results: Women who did not have the abortion had a lower mean age than those who underwent the procedure $(22.9 \pm 4.5$ years versus $25.4 \pm 7.2$ years), as well as higher mean gestational age (15.0 \pm 7.5 weeks versus $11.7 \pm 4.4$ weeks). Low schooling, no occupation, known aggressor, not performing police report and not performing a medical-legal examination was more frequent in women who did not have an abortion. Among the reasons for not performing abortion, the woman's withdrawal was the most frequent $(25.0 \%)$, followed by gestational age $\geq 23$ weeks (23.1\%). Conclusion: The advanced gestational age and the woman's withdrawal were the main reasons for not performing the legal abortion. The findings suggest that the greater vulnerability of younger women without income and with little schooling ends up as an obstacle. Known aggressors who use threats seem to exert some influence in these cases, possibly postponing the search for legal abortion.
\end{abstract}

Key words: Legal abortion, Unwanted pregnancy, Sexual violence, Violence against women, Crime victims.

\title{
1 INTRODUÇÃO
}

O aborto representa importante problema de saúde pública, particularmente para países em desenvolvimento que adotam leis proibitivas. ${ }^{1}$ Apesar da legislação brasileira ser considerada muito restritiva, o artigo 128 do Código Penal, Lei n ${ }^{\circ} 2.848$, de 1940, estabelece a permissão do aborto para evitar a morte da gestante ou nos casos de gravidez decorrente de violência sexual. Em 2012, deixou de ser crime o aborto de fetos anencéfalos, resultado de julgamento do Supremo Tribunal Federal. ${ }^{2}$ 
O Brasil é signatário da Assembleia Geral das Nações Unidas, de 1999, que estabelece que nas circunstâncias em que o aborto não conflite com a lei os serviços de saúde devem garanti-lo de forma segura e humanizada. ${ }^{3}$ A gravidez que resulta da violência sexual representa uma grave violação de direitos humanos e de direitos sexuais e reprodutivos. Por seu indiscutível amparo legal, o aborto representa um direito da mulher nesses casos, cabendo ao Estado brasileiro promover ações e políticas públicas que garantam o acesso a procedimentos adequados pelo Sistema Único de Saúde (SUS). ${ }^{4}$

Os casos de gravidez decorrente de violência sexual revelam parte da magnitude da violência contra a mulher como categoria analítica de gênero, resultado da construção política e sociocultural de papéis desiguais entre o masculino e o feminino, com o objetivo de manter subordinação e controle da mulher. A Organização Mundial da Saúde (OMS) estimou, em 2002, que 20\% das mulheres no mundo sofreram violência sexual ao menos uma vez na vida, prevalência entre $13 \%$ e $28 \% .^{5}$

No Brasil, órgãos de segurança pública registraram, em 2019, 55.811 casos de violência sexual implicando em taxa de 53,1/100.000 mulheres. ${ }^{6}$ Contudo, os registros oficiais estão distantes de expressar a realidade. Constrangimento, humilhação, medo de represálias do agressor, falta de apoio e temor da repercussão social, levam a maior parte das mulheres a não comunicar o crime sexual para as autoridades da segurança pública. ${ }^{7}$

A convergência de fenômenos complexos como a gravidez por violência sexual e o aborto traz grandes desafios para a saúde pública. Ainda que previsto na lei, mulheres brasileiras enfrentaram diferentes obstáculos para acessar o aborto nessas circunstâncias, particularmente pela escassez de serviços públicos de saúde que oferecem o procedimento, obrigando-as a recorrer ao aborto clandestino e inseguro. ${ }^{8}$ Considerando-se a pouca informação sobre o aborto em situações de gravidez decorrente de crimes sexuais em nosso meio, o objetivo deste estudo é analisar os desfechos das solicitações de aborto legal segundo as características sociodemográficas das mulheres.

\section{MÉTODO}

\subsection{DELINEAMENTO DO ESTUDO}

Estudo epidemiológico transversal realizado com amostra de conveniência obtida a partir de mulheres com alegação de gravidez decorrente de violência sexual e solicitação de aborto legal, atendidas no Hospital Pérola Byington, São Paulo, Brasil, entre agosto de 1994 e dezembro de 2017. O Hospital Pérola Byington tem gestão da Secretaria de Estado da Saúde de São Paulo e é reconhecido pelo Ministério da Saúde como uma das principais referências do país para o atendimento de mulheres em situação de violência sexual e para casos de interrupção legal da gravidez. 


\subsection{CRITÉRIOS DE SELEÇÃO E INCLUSÃO}

A população de estudo foi composta por 2.418 gestantes distribuídas em dois grupos em função da variável aborto. No primeiro grupo, foram alocadas 1.782 pacientes que realizaram o aborto legal. O segundo grupo foi constituído por 636 mulheres que não realizaram a interrupção da gravidez. Prontuários não localizados ou com erro de identificação corresponderam a 20 casos, perda de $0,8 \%$ da amostra.

A violência sexual foi caracterizada segundo a narrativa da mulher ou de seu representante legal, consistente e concordante com os artigos 213 e 217-A do Código Penal, Lei n 12.015, que trata dos Crimes Contra a Dignidade Sexual. O crime de estupro, artigo 213, considera qualquer ato sexual sem consentimento, praticado com uso da força física ou de grave ameaça. O crime de estupro de vulnerável, artigo 217-A, inclui atos sexuais contra crianças e adolescentes menores de 14 anos, ou contra pessoas vulneráveis que não possam oferecer resistência ao agressor. ${ }^{2}$

As narrativas registradas em prontuário resultaram da análise consensual de equipe interdisciplinar, composta por médico ginecologista, psicólogo e assistente social. Não foi exigido boletim de ocorrência policial, laudo de exame de corpo de delito e conjunção carnal, ou alvará judicial para a inclusão no estudo.

\subsection{VARIÁVEIS DE ESTUDO}

Consideramos variáveis sociodemográficas das mulheres que incluíram a idade, escolaridade, raça/cor, situação de união, declaração de religião e ocupação. A caracterização do crime sexual considerou a identificação do agressor, o número de agressores, o uso de ameaça grave para praticar o crime, a idade gestacional no ingresso para atendimento, e a realização de boletim de ocorrência policial e de exame médico-legal. Para a análise da escolaridade consideramos por baixa escolaridade a declaração de não ter completado o ensino fundamental, equivalente a menos de nove anos de estudo. A variável raça/cor analisou apenas mulheres brancas e negras (soma das pardas e pretas), excluindose as amarelas e indígenas. Nos casos em que o aborto não foi realizado descreveu-se o motivo registrado pela instituição.

\subsection{INSTRUMENTOS}

Os dados de interesse para o estudo foram extraídos dos prontuários hospitalares e transferidos para software Epi Info, versão 7.2.3.1. A verificação da consistência dos dados foi realizada por dupla digitação, comparação dos arquivos e correção de divergências. 


\subsection{ANÁLISE ESTATÍSTICA}

Foram construídas tabelas de frequência entre realizar ou não o aborto e as variáveis de estudo. Aplicou-se o teste de Qui-quadrado de Pearson $\left(\chi^{2}\right)$ para tabelas de contingência e associação, e o teste de Mann Whitney para amostras independentes. Fixou-se em 0,05 $(p<0,05)$ o nível de rejeição da hipótese de nulidade e Intervalo de Confiança (IC) de 95\%

\subsection{ASPECTOS ÉTICOS E LEGAIS}

Foram observadas as resoluções no 196/1996 e n 466/12 do Conselho Nacional de Saúde sobre aspectos éticos inerentes à pesquisa com seres humanos. Nenhuma paciente foi por qualquer meio identificada. O manejo das informações foi realizado em computador protegido por senha e com acesso restrito aos pesquisadores. O estudo foi aprovado pelo Comitê de Ética em Pesquisa do Hospital Pérola Byington, protocolo ${ }^{\circ}$ 046/11, com dispensa do Termo de Consentimento Livre e Esclarecido.

Crimes sexuais contra menores de 18 anos foram comunicados ao Conselho Tutelar, conforme artigo $13^{\circ}$ da Lei ${ }^{\circ}$ 8.069, de 1990. A Notificação Compulsória, Decreto-Lei 5.099, de 2004, foi realizada para todos os casos a partir da data de vigência. Para a justificação legal dos abortos foi observada a Portaria MS/GM n ${ }^{\circ} 1.508$, do Ministério da Saúde. ${ }^{8}$

\section{RESULTADOS}

Entre pacientes que realizaram o aborto a idade variou de 10-44 anos, média de 25,4 7,2 anos, mediana de 25 anos. No grupo que não realizou o aborto variou de 12-47 anos, média de 22,9 \pm 4,5 anos, mediana de 21 anos. O teste de Mann Whitney foi significante $(\mathrm{p}<0,001)$, indicando idade menor entre mulheres que não realizaram o aborto. O mesmo foi verificado pelo Qui-quadrado de Pearson quando as mulheres foram agrupadas como adolescentes ou adultas $(\mathrm{p}<0,001)$.

Na tabela 1 encontram-se os dados sociodemográficos das pacientes. Não realizar o aborto foi mais frequente entre mulheres com baixa escolaridade $(p<0,001)$ e que não tinham trabalho formal ou informal $(\mathrm{p}<0,001)$.

Tabela 1 - Características sociodemográficas de mulheres em situação de violência sexual e gravidez segundo realização ou não do aborto. Hospital Pérola Byington, 1994-2017

\begin{tabular}{|c|c|c|c|c|c|c|c|c|}
\hline & \multicolumn{2}{|c|}{$\begin{array}{c}\text { ABORTO SIM } \\
(\mathrm{n}=1.782)\end{array}$} & \multicolumn{2}{|c|}{$\begin{array}{c}\text { ABORTO NÃO } \\
(\mathrm{n}=636)\end{array}$} & \multicolumn{2}{|c|}{$\begin{array}{c}\text { TOTAL } \\
(\mathrm{n}=2.418)\end{array}$} & \multirow[b]{2}{*}{ OR (IC 95\%) } & \multirow[b]{2}{*}{$p$} \\
\hline & $\mathbf{n}$ & $\%$ & $\mathbf{n}$ & $\%$ & $\mathbf{n}$ & $\%$ & & \\
\hline \multicolumn{9}{|l|}{ Grupo etário } \\
\hline Adolescentes $(<18$ & 346 & 19,4 & 233 & 36,6 & 579 & 23,9 & \multirow{2}{*}{$\begin{array}{c}0,41(0,34- \\
n=n\end{array}$} & \multirow[t]{2}{*}{$<0,001$} \\
\hline Adultas ( $\geq 18$ anos) & 1.436 & 80,6 & 403 & 63,4 & 1.839 & 76,1 & & \\
\hline Escolaridade * & & & & & & & & \\
\hline
\end{tabular}


$<9$ anos de estudo

$\geq 9$ anos de estudo

\section{Raça/Cor **}

Branca

Negra

Situação de união ***

Sim

Não

Declaração religião $* * * *$

\begin{tabular}{|c|c|c|c|c|c|c|c|c|}
\hline Sim & 1.427 & 81,9 & 482 & 81,1 & 1.909 & 81,7 & \multirow{2}{*}{$\begin{array}{c}1,04(0,82- \\
1 \text { ? }\end{array}$} & \multirow[t]{2}{*}{0,692} \\
\hline Não & 316 & 18,1 & 112 & 18,9 & 428 & 18,3 & & \\
\hline \multicolumn{9}{|c|}{ cupação ****** } \\
\hline Sim & 853 & 48,3 & 231 & 37,0 & 1.084 & 45,3 & \multirow{2}{*}{$\begin{array}{c}1,59(1,31- \\
\mathbf{1} \cap \mathbf{1}\end{array}$} & \multirow[t]{2}{*}{$<0,001$} \\
\hline Não & 914 & 51,7 & 394 & 63,0 & 1.308 & 54,7 & & \\
\hline
\end{tabular}

* excluídos 16 casos (grupo aborto sim) e 10 casos (grupo aborto não) sem registro de escolaridade

** excluídos 63 casos de mulheres indígenas e 25 casos de amarelas (grupo aborto sim) e 15 casos de mulheres indígenas e 3 casos de amarelas (grupo aborto não).

*** excluídos 58 casos (grupo aborto sim) e 39 casos (grupo aborto não) sem registro de situação conjugal.

**** excluídos 39 casos (grupo aborto sim) e 42 casos (grupo aborto não) sem registro de declaração de religião.

***** excluídos 15 casos (grupo aborto sim) e 11 casos (grupo aborto não) sem registro de ocupação.

Na tabela 2 encontram-se dados sobre o crime sexual. Agressores desconhecidos prevaleceram nos dois grupos, mas com maior frequência entre mulheres que realizaram o aborto $(\mathrm{p}<0,001)$. Nas mulheres que não interromperam a gravidez observou-se uso mais frequente de ameaça grave e menor realização do boletim de ocorrência policial e do exame médico-legal.

Tabela 2 - Características da violência sexual e procedimentos legais adotados por mulheres em situação de violência sexual e gravidez segundo realização ou não do aborto. Hospital Pérola Byington, 1994-2017

\begin{tabular}{|c|c|c|c|c|c|c|c|c|}
\hline & \multicolumn{2}{|c|}{$\begin{array}{c}\text { ABORTO SIM } \\
(\mathrm{n}=1.782)\end{array}$} & \multicolumn{2}{|c|}{$\begin{array}{c}\text { ABORTO NÃO } \\
(n=636)\end{array}$} & \multicolumn{2}{|c|}{$\begin{array}{c}\text { TOTAL } \\
(\mathrm{n}=2.418)\end{array}$} & \multirow[b]{2}{*}{ OR } & \multirow[b]{2}{*}{$p$} \\
\hline & $\mathbf{n}$ & $\%$ & $\mathbf{n}$ & $\%$ & $\mathbf{n}$ & $\%$ & & \\
\hline \multicolumn{9}{|c|}{ Autor da violência * } \\
\hline Desconhecido & 1.229 & 69,2 & 359 & 56,8 & 1.588 & 66,0 & \multirow{2}{*}{$\begin{array}{c}1,10(1,41- \\
\text { ก กะ }\end{array}$} & \multirow[t]{2}{*}{$<0,001$} \\
\hline Conhecido & 547 & 30,8 & 273 & 43,2 & 820 & 34,0 & & \\
\hline \multicolumn{9}{|c|}{ Número de agressores ** } \\
\hline Único & 1.611 & 92,0 & 577 & 91,6 & 2.188 & 91,9 & \multirow{2}{*}{$\begin{aligned} 1,05 & (0,79- \\
& 1 \text { 17 }\end{aligned}$} & \multirow[t]{2}{*}{0,742} \\
\hline Múltiplo & 140 & 8,0 & 53 & 8,4 & 193 & 8,1 & & \\
\hline \multicolumn{9}{|l|}{ Ameaça grave } \\
\hline Sim & 277 & 15,5 & 248 & 39,0 & 525 & 21,7 & \multirow[t]{2}{*}{$\begin{array}{l}0,28(0,23- \\
0,35)\end{array}$} & \multirow[t]{2}{*}{$<0,001$} \\
\hline Não & 1.505 & 84,5 & 388 & 61,0 & 1.893 & 78,3 & & \\
\hline \multicolumn{9}{|c|}{ Boletim de ocorrência $* * *$} \\
\hline Sim & 939 & 53,0 & 403 & 64,4 & 1.342 & 56,0 & $0,62(0,51-$ & $<0,001$ \\
\hline
\end{tabular}




$\begin{array}{lllllll}\text { Não } & 833 & 47,0 & 223 & 35,6 & 1.056 & 44,0\end{array}$

Exame medico-legal $* * * *$

$\begin{array}{lllllllll}\text { Sim } & 869 & 49,8 & 366 & 59,7 & 1.235 & 52,4 & \begin{array}{c}0,67(0,55- \\ \text { ก on }\end{array} \\ \text { Não } & 875 & 50,2 & 247 & 40,3 & 1.122 & 47,6 & & <0,001\end{array}$

* excluídos 6 casos (grupo aborto sim) e 1 caso (grupo aborto não) sem registro da identificação do agressor

** excluídos 26 casos (grupo aborto sim) e 6 casos (grupo aborto não) sem registro do número de agressores

*** excluídos 10 casos (grupo aborto sim) e 10 casos (grupo aborto não) sem dados sobre de boletim de ocorrência

**** excluídos 38 casos (grupo aborto sim) e 23 casos (grupo aborto não) sem dados sobre exame médico-legal

No grupo que realizou o aborto legal a idade gestacional variou de 5-22 semanas, média de 11,7 $\pm 4,4$ semanas. No grupo que não realizou a interrupção da gravidez a idade gestacional variou de 437 semanas com média de 15,0 \pm 7,5 semanas. Mesmo excluindo-se os casos com idade gestacional $\geq$ 23 semanas no grupo que não realizou o aborto, a diferença se manteve significante $(\mathrm{p}=0,021)$, com maior média de idade gestacional entre mulheres que não realizaram o procedimento.

Em 636 casos a solicitação do aborto legal não foi atendida (Tabela 3). Entre os motivos, destacam-se a desistência da mulher após receber parecer institucional favorável $(25,0 \%)$ e a idade gestacional $\geq 23$ semanas ou estimativa de peso fetal $>500$ gramas $(23,1 \%)$.

Tabela 3 - Motivo para não realização do aborto de 636 mulheres em situação de violência sexual e gravidez. Hospital Pérola Byington, 1994-2017

\begin{tabular}{lcc}
\hline Motivo & n & \% \\
\hline Desistência do aborto após aprovação institucional & 159 & 25,0 \\
Idade gestacional $\geq 23$ semanas / peso fetal $>$ 500 gramas & 147 & 23,1 \\
Gravidez prévia ou posterior à violência sexual & 113 & 17,8 \\
Perda de acompanhamento & 87 & 13,7 \\
Narrativa discordante do artigo 213 ou 217-A do Código Penal & 65 & 10,2 \\
Impossibilidade de exclusão de paternidade do parceiro íntimo & 24 & 3,8 \\
Paternidade do parceiro íntimo determinada por DNA fetal & 6 & 0,9 \\
Outro & 35 & 5,5 \\
\hline
\end{tabular}

\section{DISCUSSÃO}

A gravidez indesejada e forçada pela agressão sexual é entendida pela maioria das mulheres como uma segunda expressão da violência ${ }^{9}$, o que as mobiliza na busca legítima pelo aborto legal ${ }^{10}$. As evidências indicam que crimes sexuais são mais prevalentes entre mulheres mais jovens. ${ }^{4,11,12,13} \mathrm{~A}$ pouca idade da vítima se mostra ainda mais importante quando há outras vulnerabilidades envolvidas no crime sexual, como ocorre entre as deficientes intelectuais ${ }^{14}$ ou nas situações de incesto ${ }^{4}$.

Em 2109, os órgãos de segurança pública brasileiros indicaram que quase $60 \%$ dos registros de violência sexual ocorreram entre menores de 13 anos. ${ }^{6}$ Outro estudo brasileiro constatou quase $25 \%$ 
dos casos de violência sexual contra adolescentes de 12 a 17 anos. ${ }^{15}$ Análise de notificações compulsórias, realizada em Santa Catarina, Brasil, observou que a idade entre 10 e 14 anos mostrou chance três vezes maior de se associar com gravidez decorrente da violência sexual. ${ }^{16}$ Em nossos resultados, observamos que a média de idade foi menor entre as mulheres que não realizou o aborto legal.

Pesquisas também indicam relação entre baixa escolaridade e crimes sexuais, tanto praticados por desconhecidos ${ }^{12,17,18}$ como por parceiros íntimos ${ }^{19,20}$. Em nossos resultados, parte relevante das participantes apresentou menos do que nove anos de estudo (27,2\%), compatível com o relatório do Programa das Nações Unidas para o Desenvolvimento, de 2018, no qual o Brasil aparece com média de escolaridade de 7,8 anos. $^{21}$

No cenário brasileiro, maiores taxas de aborto induzido são descritas entre mulheres com baixa escolaridade. $^{13,22}$ A Pesquisa Nacional de Aborto, de 2016, indica que 38\% das mulheres brasileiras que recorre ao aborto clandestino tem menos do que nove anos de estudo. ${ }^{22}$ Neste estudo, o percentual de mulheres com baixa escolaridade $(27,8 \%)$ foi pouco menor do que o apontado pelos dados nacionais. Contudo, são situações distintas quanto a legalidade do aborto. Além disso, a população incluída neste estudo foi de mulheres da região metropolitana de São Paulo, com melhores indicadores de escolaridade. Mesmo assim, a baixa escolaridade se mostrou mais frequente entre mulheres que não chegaram a realizar o aborto legal.

A raça/cor se mostra outro elemento que perpassa a violência e os conflitos de gênero. O último censo brasileiro aponta que $47,7 \%$ da população se autodeclara branca ${ }^{23}$, semelhante ao observado em estudos com mulheres que buscam atendimento nos serviços de saúde após sofrerem crimes sexuais $^{13,24}$, ou que recorrem ao aborto legal nessas circunstâncias ${ }^{4,10,12}$.

O Instituto Brasileiro de Geografia e Estatística (2016) ${ }^{23}$, alerta que as mulheres negras brasileiras constituem grupo de grande vulnerabilidade para sofrer violências. Pesquisa com base nas informações do Sistema de Vigilância de Acidentes e Violências aponta que $70 \%$ dos registros de violência envolve mulheres negras. ${ }^{25}$ Em amostra representativa domiciliar observou-se que mulheres negras brasileiras têm maior necessidade de recorrer ao aborto. ${ }^{22}$ Nos casos de violência sexual praticada pelo parceiro íntimo, pesquisa em Salvador, Brasil, indica relação relevante entre gravidez indesejada e aborto clandestino. ${ }^{26}$ Esse recorte de desigualdade de saúde reprodutiva se repete em outros países, como nos EUA, em que a taxa de aborto é cinco vezes maior entre mulheres negras. ${ }^{27}$

Considerando esses aspectos, excluímos 106 casos (4,4\% da amostra) de mulheres que se declararam amarelas ou indígenas, permitindo verificar a situação de mulheres negras que buscam o aborto legal. Divergindo da maioria das evidências, não constatamos diferença no desfecho do aborto legal segundo raça/cor. 
Neste estudo, a maioria das mulheres reportou ser solteira ou sem união em ambos os grupos, informação igualmente constatada em outros estudos brasileiros com mulheres em situação de violência sexual. ${ }^{12,13,28}$ As repercussões emocionais e comportamentais do parceiro íntimo nas situações de violência sexual são pouco reportadas. Ahrens e Aldana (2012) ${ }^{29}$ observaram que enquanto parte das relações afetivas se fortaleceu após as mulheres compartilharem o ocorrido com o parceiro íntimo, outras afirmaram que houve evidente deterioramento nas relações interpessoais. Dessa forma, entendemos que a situação de união não implica necessariamente em apoio do parceiro que a mulher realize o aborto legal. Não verificamos neste estudo diferença na interrupção da gravidez entre mulheres segundo situação de união, embora não tenha sido possível conhecer se as mulheres estudadas revelaram a violência sexual ou a gravidez para seus parceiros.

Em nossos resultados, a maioria das participantes declarou alguma religião, permitindo inferir que suas crenças não foram obstáculo para que recorressem ao aborto legal, como observado em nosso meio por Blake et al. $(2015)^{12}$ e por Pimentel et al. $(2020)^{30}$. Contudo, a questão do aborto permanece complexa, particularmente para a igreja católica e neopentecostais, com posições axiomáticas mesmo nas circunstâncias de violência sexual. ${ }^{10,30}$

Ao mesmo tempo, vivências religiosas podem se relacionar com diferentes formas de violência contra a mulher. No Brasil, há evidências de que mulheres evangélicas sofram mais violência física e psicológica dentro do casamento do que as católicas. ${ }^{24} \mathrm{Na}$ Índia, mulheres afiliadas ao cristianismo têm menor risco de sofrer violência pelo parceiro íntimo. ${ }^{19}$ No Iraque, observou-se maior prevalência de violência física, sexual e psicológica entre mulheres muçulmanas do que em cristãs. ${ }^{31}$

Há pouca informação sobre o papel das religiões no aborto de gestações decorrentes de violência sexual. ${ }^{30}$ Neste estudo, não observamos diferença na realização do aborto segundo declaração de religião. Mas é necessário observar que analisamos somente mulheres que buscaram um serviço de aborto legal, não sendo possível conhecer se a religião influenciou aquelas que decidiram manter a gravidez até o termo. Além disso, o desenho do estudo não permite afirmar que a religião tenha exercido papel nas desistências do aborto observadas.

A violência também atinge com maior frequência mulheres sem ocupação remunerada ${ }^{25}$, dando significado para violência de gênero numa perspectiva de estratificação social. ${ }^{32}$ Estudo no Nepal constatou que a maior autonomia econômica da mulher reduz o risco de sofrer violência sexual. ${ }^{33}$ Nos EUA, a baixa renda da mulher se associou com maior risco de sofrer violência sexual nas relações domésticas. ${ }^{34}$

Embora não tenhamos identificado estudos abordando a relação entre ocupação ou renda em situações de aborto legal, constatamos que a interrupção da gravidez foi menor entre mulheres sem ocupação. Embora se suponha que a falta de ocupação implique em menor autonomia econômica, o 
banco de dados não permitiu estabelecer criério de renda que pudesse adequadamente analisar este achado.

Crimes sexuais geralmente são praticados por agressores conhecidos ${ }^{5}$, principalmente quando se trata de crianças e adolescentes ${ }^{35}$. Estudo promovido pela OMS encontrou que mulheres com histórico de violência pelo parceiro íntimo apresentam chance significativamente maior de gravidez não planejada e de recorrer ao aborto. ${ }^{36}$ Em pesquisa com base populacional na Tanzânia, mulheres em situação de violência física e sexual pelo parceiro íntimo mostraram chance 1,9 vezes maior de aborto induzido. ${ }^{37}$

No Brasil, a busca por atendimento nos serviços de emergência após casos de violência sexual aponta para o predomínio de perpetradores desconhecidos ${ }^{4,10,13,35}$, sugerindo que ainda existem obstáculos para que as mulheres percebam ou revelem violências praticadas por pessoas próximas, principalmente o parceiro íntimo ${ }^{12,17,24,30}$ Neste estudo, nos casos em que o aborto não foi realizado observamos maior frequência de agressores conhecidos. A quantificação dos agressores também importa pelas consequências psicológicas mais severas que determina para as mulheres ou pelo maior risco de infecções sexualmente transmissíveis (IST). ${ }^{8,10}$ Em 193 casos $(8,1 \%)$ constatamos que a violência sexual ocorreu com a participação de dois ou mais agressores, embora sem diferença nos dois grupos.

O uso da violência física pelo agressor nos crimes sexuais pode mostrar efeitos impactantes. Na África do Sul, estudo de âmbito nacional identificou que 19,8\% dos feminicídios foram precedidos de violência sexual. ${ }^{38}$ Nos EUA, entre os anos de 2004 e 2006, registrou-se 105 mil atendimentos de emergência para tratamento de lesões decorrentes da violência sexual contra mulheres jovens. ${ }^{39}$ Riggs et al. (2000) $)^{40}$ observaram lesões genitais em metade das mulheres americanas que sofreram violência sexual por desconhecidos que empregaram a violência física.

Apesar da importância da violência física nos crimes sexuais, a grave ameaça, incluindo-se a promessa de morte, é descrita como o principal recurso intimidatório do agressor para praticar a violência sexual em nosso meio. ${ }^{4,10,13}$ De fato, constatamos neste estudo que entre mulheres que não realizaram o aborto foi mais frequente sofrer violência sexual mediante grave ameaça. Além disso, o estudo das rotas e trajetórias das mulheres brasileiras que sofrem violência sexual sustenta que a maioria não recorre aos serviços de saúde e não comunica o crime para a polícia. ${ }^{4,10,12,24,35}$

Quando a violência sexual termina em gravidez, o ordenamento jurídico brasileiro garante que a realização do aborto não se condiciona à apresentação de alvará judicial, boletim de ocorrência policial ou exame médico-legal. ${ }^{2,8}$ Embora quase a metade das mulheres neste estudo tenha realizado os procedimentos policiais e exame no Instituto Médico Legal (IML), a apresentação desses documentos foi significativamente maior nos casos em que o aborto legal não foi realizado. 
A recusa dos procedimentos e cuidados disponíveis pode se relacionar com a proximidade com o agressor, tornando as mulheres mais sujeitas ao constrangimento, intimidação ou ameaça. ${ }^{4,10,12}$ Também é possível que elementos da cultura patriarcal, tolerante com a violência, possam desmobilizar as mulheres ou reduzir sua percepção sobre seus direitos. ${ }^{5,9,41}$ As desigualdades nas relações de gênero naturalizam e invisibilizam a violência contra a mulher, tornando implícita a ideia de subalternidade. ${ }^{41}$ No mesmo sentido, o trauma emocional pode interferir no reconhecimento dos riscos para a saúde. ${ }^{42}$ Parte expressiva das mulheres teme receber tratamento inquisitório ou julgador, ou que o serviço de saúde revele a violência sexual para a polícia contra seu desejo. ${ }^{41}$

O agressor sexual conhecido ou próximo da mulher parece ser o elemento mais importante relacionado aos casos em que o aborto não foi realizado, seja por desistência ou por idade gestacional avançada. Em nosso meio, Pimentel et al. $(2020)^{30}$ constataram que a chance de desistir do aborto legal foi duas vezes maior quando a vítima conhecia o agressor. Para os autores, idade, religião, escolaridade, raça/cor, situação de união e tipo de intimidação não se associaram com a desistência do aborto legal. ${ }^{30}$

Neste estudo, o principal motivo para não realizar o aborto foi a desistência da mulher, em 159 casos (25,0\%). Contudo, houve perda do acompanhamento em 87 casos (13,7\%), sendo possível supor que parte dessas mulheres também tenha desistido do procedimento não retornando para atendimento. Mas, perda gestacional espontânea ou recorrer a outro serviço de saúde de saúde podem ter ocorrido entre esses casos.

Foster e Kimport (2013) ${ }^{43}$ encontraram que conflitos com o parceiro íntimo estavam entre os fatores para a busca do aborto em idade gestacional tardia. Achado semelhante foi observado por Blake et al. $(2015)^{12}$ e por Perry et al. (2016) ${ }^{44}$, verificando que agressores sexuais conhecidos se associaram com expressiva postergação da busca pelo aborto legal, resultando em maior idade gestacional.

O mesmo ocorreu no estudo de Bessa et al. (2019) ${ }^{4}$, analisando casos de incesto de adolescentes. Nesses casos, a idade pode refletir maior dificuldade das mulheres mais jovens em acessar os serviços de saúde, menor autonomia nas decisões sobre seu futuro reprodutivo, menor suporte social ou familiar, ou pela percepção mais tardia da gravidez. ${ }^{45}$

Por sua vez, a idade gestacional mais avançada termina como limitador do aborto legal. Embora a legislação brasileira não restrinja o conceito jurídico do aborto segundo a idade gestacional ${ }^{2}$, a OMS estabelece limite baseado na inviabilidade fetal, tendo como orientação a $22^{a}$ semana, quando o peso fetal não excede 500 gramas. ${ }^{1}$ A normativa brasileira vigente sobre aborto em casos de violência sexual adota esses parâmetros. ${ }^{8}$ Entre mulheres brasileiras, Mutta e Yela (2017) ${ }^{13}$ encontraram 63,4\% das solicitações de aborto legal com menos de 13 semanas de idade gestacional. Madeiro e Diniz $(2016)^{28}$, verificaram que a idade gestacional entre 9 e 14 semanas foi mais comum na busca do aborto. Neste 
estudo, os casos que realizaram o aborto apresentaram menor idade gestacional, com média de 11,7 semanas.

Neste estudo, entre as mulheres que não realizaram o aborto, encontramos 147 casos $(23,1 \%)$ limitados pela idade gestacional $\geq 23$ semanas. Semelhante aos nossos resultados, regressão multinominal realizada por Blake et al $(2015)^{12}$ mostrou que a busca tardia pelo aborto legal foi mais frequente entre mulheres mais jovens que sofreram violência por agressores conhecidos ou aparentados, com maior comunicação do ocorrido para a polícia. Divergindo de nossos achados, os autores não encontraram associação com a escolaridade ou situação conjugal. ${ }^{12}$

Em 113 casos (17,8\%) não se aprovou a solicitação por se tratar de gravidez prévia ou posterior à violência sexual. Essas situações foram definidas em função de diferença significativa entre a idade gestacional mensurada por duas ultrassonografias obstétricas e a idade gestacional com base na data da violência sexual conhecida e de certeza. Em outros 65 casos (10,2\%), a narrativa apresentada pela mulher ou por seu representante legal se mostrou claramente discordante dos limites definidos pelos artigos 213 ou 217-A, não oferecendo fundamento legal para o aborto.

Em 30 casos $(4,7 \%)$ a violência sexual ocorreu em data próxima de relação sexual consentida com parceiro não agressor, impossibilitando estabelecer, por critérios clínicos, se a gravidez decorria de violência sexual. Em seis casos $(0,9 \%)$ análise de DNA fetal intraútero concluiu pela paternidade do parceiro. Nos outros 24 casos $(3,8 \%)$ o exame não foi realizado por falta de acessibilidade ao método ou desistência da mulher em fazê-lo.

Os resultados deste estudo podem contribuir para o entendimento do fenômeno da violência contra a mulher, particularmente quanto a responsabilidade ética dos profissionais de saúde. ${ }^{46,47} \mathrm{~A}$ gravidez forçada e o aborto nessas circunstâncias são questões relevantes para a saúde pública, principalmente nos países que mantém legislações restritivas ou proibitivas. Os pontos fortes deste estudo são sua originalidade, seu aspecto documental com 23 anos de atendimentos em uma instituição de referência, assim como número de casos analisados.

As limitações deste estudo devem ser observadas, como o uso de amostra de conveniência. Cabe cuidado para não generalizar os resultados para outros contextos sociais e culturais. Restrição de validade externa deve ser observada para variáveis como raça/cor e religião, devido possível heterogeneidade sociodemográfica de outras amostras. As trajetórias das vítimas nas instituições e os procedimentos legais também estão sujeitos a essa restrição, considerando-se a diversidade das legislações sobre crimes sexuais e aborto em outros países. Admitimos eventual imprecisão nas respostas das mulheres, principalmente nas variáveis como a identidade do agressor e forma de intimidação, devido o temor de represálias. 


\section{CONCLUSÃO}

Não realizar o aborto legal não se relacionou com a raça/cor, situação de união ou declaração de religião da mulher, ou com a imposição do crime sexual por meio de violência física. A idade gestacional avançada e a desistência da mulher após a aprovação da solicitação foram as principais razões para não realizar a interrupção da gravidez. Os achados sugerem que a maior vulnerabilidade de mulheres mais jovens, sem renda e com pouca escolaridade termine como obstáculo para acessar o aborto. Agressores conhecidos e que utilizam de ameaças parecem exercer influência nesses casos, possivelmente postergando a busca pelo aborto legal. 


\section{REFERÊNCIAS}

World Health Organization. Safe abortion: Technical and policy guidance for health systems. 2th edition. Geneva: World Health Organization; 2012. 132p.

Masson C. Código Penal Comentado. $7^{\text {a }}$ ed. São Paulo: Método; 2019. 1504p.

United Nations. Key actions for the further implementation of the Programme of Action of the International Conference on Population and Development. New York: United Nations; 1999.

Bessa MMM, Drezett J, Adami F, Araújo SDT, Bezerra IMP, Abreu LC. Characterization of adolescent pregnancy and legal abortion in situations involving incest or sexual violence by an unknown aggressor. Medicina (Kaunas). 2019; 55 (8): 474. DOI: 10.3390/medicina55080474.

Krug EG, Dahlberg LL, Mercy JA, Zwi AB, Lozano R. World report on violence and health. Geneva: World Health Organization; 2002. 346p.

Fórum Brasileiro de Segurança Pública. Anuário brasileiro de segurança pública 2020. 14ª ed. Brasília: Fórum Brasileiro de Segurança Pública; 2020. 332p.

Pilleco FB, Knauth DR, Vigo Á. Sexual coercion and abortion: a context of vulnerability among youth women. Cad Saude Publica. 2011, 27 (3): 427-39. DOI: 10.1590/S0102-311X2011000300004.

Brasil. Ministério da Saúde. Secretaria de Atenção à Saúde. Departamento de Ações Programáticas Estratégicas. Prevention and treatment of damages resulting from sexual violence against women and adolescents: technical standard. $3^{\mathrm{a}}$ ed. Brasília: Editora do Ministério da Saúde; 2012. 124p.

Faúndes A, Barzelatto J (editors). O drama do aborto. Em busca de um consenso. Campinas: Komedi; 2004.

Drezett J, Pedroso D, Vertamatti MA, Macedo-Júnior H, Blake MT, Gebrim LH, et al. Pregnancy resulting from sexual abuse: reasons alleged by Brazilian women for carrying out the abortion pregnancy and violence. HealthMED. 2012; 6 (3): 819-25.

Avegno J, Mills TJ, Mills LD. Sexual assault victims in the emergency department: analysis by demographic and event characteristics. J Emerg Med. 2009; 37 (3): 328-34. DOI: 10.1016/j.jemermed.2007.10.025.

Blake MT, Drezett J, Machi GS, Pereira VX, Raimundo RD, Oliveira FR, et al. Factors associated with the delay in seeking legal abortion for pregnancy resulting from rape. Int Arch Med. 2015; 8 (29): 114. DOI: $10.3823 / 1628$.

Mutta DS, Yela DA. Sociodemographic characteristics of women in a public hospital in Campinas who underwent legal abortion due to sexual violence: cross-sectional study. Sao Paulo Med J. 2017; 135 (4): 363-8.

Brunnberg E, Boström ML, Berglund M. Sexual force at sexual debut. Swedish adolescents with disabilities at higher risk than adolescents without disabilities. Child Abuse Negl. 2012; 36 (4): 285 95. DOI: 10.1016/j.chiabu.2012.01.002. 
Waiselfisz JJ. Mapa da Violência 2015: Homicídio de Mulheres no Brasil. Brasília: FLACSO; 2015. 83p.

Delziovo CR, Coelho EBS, D'Orsi E, Lindner SR. Sexual violence against women and care in the health sector in Santa Catarina - Brazil. Cienc Saude Coletiva. 2018; 23 (5): 1687-96. DOI: 10.1590/1413-81232018235.20112016.

Facuri CO, Fernandes MAS, Oliveira KD, Andrade TS, Azevedo RCS. Violência sexual: estudo descritivo sobre as vítimas e o atendimento em um serviço universitário de referência no Estado de São Paulo, Brasil. Cad Saude Publica. 2013; 29 (5): 889-98. DOI: 10.1590/S0102-311X2013000500008.

Mukanangana F, Moyo S, Zvoushe A, Rusinga O. Gender based violence and its effects on women's reproductive health: The case of Hatcliffe, Harare, Zimbabwe. Afr J Reprod Health. 2014; 18 (1): 110 22.

Kimuna SR, Djamba YK, Ciciurkaite G, Cherukuri S. Domestic violence in India: insights from the 2005-2006 national family health survey. J Interpers Violence. 2013; 28 (4): 773-807. DOI: $10.1177 / 0886260512455867$.

Halpern-Meekin S, Costanzo M, Ehrenthal D, Rhoades G. Intimate partner violence screening in the prenatal period: variation by State, insurance, and patient characteristics. Matern Child Health J. 2019; 23 (6): 756-67. DOI: 10.1007/s10995-018-2692-x.

United Nations Development Programme. Human development indices and indicators 2018. Statistical Update. Washington DC: United Nations Development Programme; 2018. 123p.

Diniz D, Medeiros M, Madeiro A. National abortion survey 2016. Cienc Saude Coletiva. 2017; 22 (2): 653-60. DOI: $10.1590 / 1413-81232017222.23812016$.

Instituto Brasileiro de Geografia e Estatística (IBGE). Ministério do Planejamento, Desenvolvimento e Gestão. Síntese de indicadores sociais: uma análise das condições de vida da população brasileira 2016. Rio de Janeiro: Instituto Brasileiro de Geografia e Estatística; 2016. 141p.

Leite FMC, Amorim MHC, Wehrmeister FC, Gigante DP. Violência contra a mulher em Vitória, Espírito Santo, Brasil. Rev Saude Publica. 2017; 51 (33): 1-12. DOI: 10.1590/s15188787.2017051006815.

Garcia LP, Silva GDM. Violência por parceiro íntimo: perfil dos atendimentos em serviços de urgência e emergência nas capitais dos estados brasileiros. Cad Saude Publica. 2018; 34 (4): e00062317. DOI: 10.1590/0102-311x00062317.

Diniz NMF, Gesteira SMA, Lopes RLM, Mota RS, Pérez BAG, Gomes NP. Voluntary abortion and domestic violence among women attended at a public maternity hospital of Salvador - BA. Rev Bras Enferm. 2011; 64 (6): 1010-15. DOI: 10.1590/s0034-71672011000600004.

Cohen SA. Abortion and women of color: the bigger picture. Alan Guttmacher Institute, 2008; 11 (3): $2-12$.

Madeiro A, Diniz D. Legal abortion services in Brazil - a national study. Cienc Saude Coletiva. 2016; 21 (2): 563-72. DOI: 10.1590/1413-81232015212.10352015. 
Ahrens CE, Aldana E. The ties that bind: understanding the impact of sexual assault disclosure on survivors' relationships with friends, family, and partners. Trauma Dissociation. 2012; 13 (2): 226-43. DOI:10.1080/15299732.2012.642738.

Pimentel RMM, Drezett J, Macedo Jr H, Andreoni S, Silva RS. Abortion withdrawal of sexual violence pregnancy: the role of the sex offender. J Hum Growth. 2020; 30 (2): 170-8. DOI: https://doi.org/10.7322/jhgd.v30.10363.

Al-Tawil NG. Association of violence against women with religion and culture in Erbil Iraq: A crosssectional study. BMC Public Health. 2012; 12: 800. DOI: 10.1186/1471-2458-12-800.

Schraiber LB, D'Oliveira AFPL, França-Junior I. Intimate partner sexual violence among men and women in urban Brazil, 2005. Rev Saude Publica. 2008; 42 (supl1): 127-37.

Puri M, Frost M, Tamang J, Lamichhane P, Shah I. The prevalence and determinants of sexual violence against young married women by husbands in rural Nepal. BMC Res Notes. 2012; 5: 291. DOI: $\underline{10.1186 / 1756-0500-5-291 .}$.

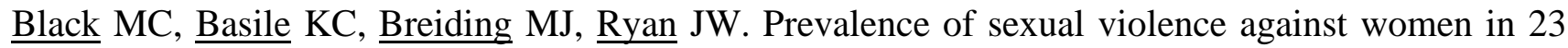
States and Two U.S. Territories, BRFSS 2005. Violence Against Women. 2014; 20 (5): 485-99. DOI: $10.1177 / 1077801214528856$.

Blake MT, Drezett J, Vertamatti MA, Adami F, Valenti VE, Paiva AC, et al. Characteristics of sexual violence against adolescent girls and adult women. BMC Women's Health. 2014; 14:15. DOI: $\underline{10.1186 / 1472-6874-14-15}$.

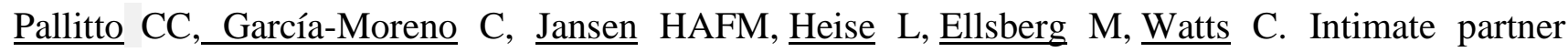
violence, abortion, and unintended pregnancy: Results from the WHO Multi-country Study on Women's Health and Domestic Violence. Int J Gynaecol Obstet. 2013; 120 (1): 3-9. DOI: 10.1016/j.ijgo.2012.07.003.

Stöckl H, Filippi V, Watts C, Mbwambo JKK. Induced abortion, pregnancy loss and intimate partner violence in Tanzania: A population based study. BMC Pregnancy Childbirth. 2012; 12: 12. DOI: 10.1186/1471-2393-12-12.

Abrahams N, Mathews S, Lombard C, Martin LJ, Jewkes R. Sexual homicides in South Africa: A national cross-sectional epidemiological study of adult women and children. PLoS One. 2017;12(10):e0186432. DOI: 10.1371/journal.pone.0186432.

Gavin L, MacKay AP, Brown K, Harrier S, Ventura SJ, Kann L, et al. Sexual and reproductive health of persons aged 10-24 years - United States, 2002-2007. MMWR Surveill Summ. 2009; 58 (6): 1-58.

Riggs N, Houry D, Long G, Markovchick V, Feldhaus KM. Analysis of 1,076 cases of sexual assault. Ann Emerg Med. 2000; 35 (4): 358-62. DOI: 10.1016/s0196-0644(00)70054-0.

Santos DLA. Mulheres na busca pelo aborto legal: rota crítica percorrida e necessidades em saúde suscitadas [tese]. São Paulo: Universidade de São Paulo; 2019. 
Souza FBC, Drezett J, Meirelles AC, Ramos DG. Aspectos psicológicos de mulheres que sofrem violência sexual. Reprod Clim. 2012; 27 (3): 98-103. DOI: 10.1016/j.recli.2013.03.002.

Foster DG, Kimport K. Who seeks abortions at or after 20 weeks? Perspect Sex Reprod Health. 2013; 45 (4): 210-8. DOI: 10.1363/4521013.

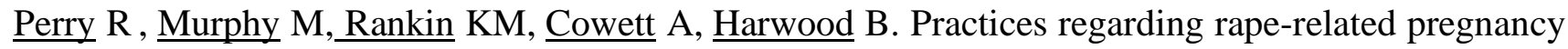
in U.S. Abortion Care Settings. Womens Health Issues. 2016; 26 (1): 67-73. DOI: 10.1016/j.whi.2015.10.006.

Grams AC, Carneiro de Sousa, MJ, Roesch R, Pinto de Costa J. Sexual abuse within the marital relationship. Med Law. 1997; 16 (4): 743-51.

Silva EP, Gaspar RB, Ferreira FG, Almeida VM, Silva JD, Veiga PS, Pereira IS, Oliveira CP. Implantação de comissão de ética de enfermagem institucional: buscando a prática com equidade. Latin American Journal of Development. 2021;3(3):1237-47. DOI: https://doi.org/10.46814/lajdv3n3-018.

Galli B, Drezett J, Cavagna-Neto M. Aborto e objecão de consciência. Cienc Cult. 2012; 64 (2): 32-5. DOI: http://dx.doi.org/10.21800/S0009-67252012000200014. 